\title{
Single crystal EPR study of VO(II)-doped cadmium potassium phosphate hexahydrate: A substitutional incorporation
}

\author{
I SOUGANDI, T M RAJENDIRAN, R VENKATESAN and \\ P SAMBASIVA RAO* \\ Department of Chemistry, Pondicherry University, R V Nagar, Kalapet, \\ Pondicherry 605 014, India \\ e-mail: psr52in@yahoo.co.in
}

MS received 16 March 2002; revised 6 September 2002

\begin{abstract}
Single crystal EPR studies of VO(II)-doped cadmium potassium phosphate hexahydrate $(\mathrm{CPPH})$ have been carried out at room temperature. The angular variation spectra in the three orthogonal planes indicate that the paramagnetic impurity has entered the lattice only substitutionally in place of $\mathrm{Cd}$ (II). Spin Hamiltonian parameters have been obtained from single crystal data. Powder spectra show a set of eight parallel and perpendicular features indicating the presence of only one site. The admixture coefficients have been calculated from the data, which agree well with the literature values.
\end{abstract}

Keywords. EPR; VO(II); cadmium potassium phosphate hexahydrate, substitutional incorporation; spin-Hamiltonian parameters.

\section{Introduction}

Vanadyl ion $\left(\mathrm{VO}^{2+}\right)$ is the most stable and widely used EPR probe for probing the site symmetry of the central metal ion and the nature of the bonding with the ligands and this has also been used as an impurity for understanding the defect, structural, phase transition and orientation properties of the host lattice ${ }^{1-5}$. Studies of VO(II) in glasses and other systems have attracted attention very recently ${ }^{6-8}$. Vanadyl ion with $3 d^{1}$ configuration allows paramagnetic resonance to be observed at ambient temperatures and hence many studies have been reported ${ }^{9-16}$. The interesting behaviour of the VO(II) ion in different crystalline matrices is due to the different orientations of the $\mathrm{V}=\mathrm{O}$ ion. In one of the previous communications dealing with $\mathrm{VO}$ (II) in zinc ammonium sulphate hexahydrate, Tutton's salt (ZASH) ${ }^{1}$, the authors have reported three types of vanadyl centres with intensity ratios of 20:5:1. However, spin Hamiltonian parameters were given only for the substitutional one, which has the highest intensity. They also mentioned that the other two low intensity sites also have similar spin Hamiltonian parameters. In addition, an extensive report on EPR study of $\mathrm{VO}(\mathrm{II})$ in $\mathrm{K}_{2} \mathrm{Zn}\left(\mathrm{SO}_{4}\right)_{2} \cdot 6 \mathrm{H}_{2} \mathrm{O}$ and $\mathrm{K}_{2} \mathrm{Mg}\left(\mathrm{SO}_{4}\right)_{2} \cdot 6 \mathrm{H}_{2} \mathrm{O}$ indicated that the impurity entered divalent metal sites and preferentially oriented in the direction of water molecules ${ }^{9}$. In the case of $\mathrm{K}_{2} \mathrm{Zn}\left(\mathrm{SO}_{4}\right)_{2} \cdot 6 \mathrm{H}_{2} \mathrm{O}$, three sites of $\mathrm{VO}$ (II) ions were found with intensity ratios of 5.0:3.75:0.2 and the directions of the $g$ tensors were collaborated with metal-water distances in the lattice. However, in case of VO(II) in

*For correspondence 
magnesium potassium sulphate hexahydrate (MPSH) ${ }^{15}$ and magnesium ammonium phosphate hexahydrate (MAPH) ${ }^{16}$, only two types of vanadyl centers were reported, corresponding to substitutional and interstitial locations. Hence, in order to understand the nature and position of the impurity, we have studied the EPR spectra of VO(II) in yet another host lattice, i.e., cadmium potassium phosphate hexahydrate (CPPH) and the results are reported in this communication.

\section{Experimental}

Single crystals of cadmium potassium phosphate hexahydrate doped with VO(II) were grown by slow evaporation of a saturated solution containing equimolar amounts of cadmium sulphate and potassium dihydrogen phosphate. To this, a small amount of the paramagnetic impurity is added in the form of vanadyl sulphate. Good blue coloured crystals were obtained within a fortnight. EPR spectra were recorded on a JEOL JESTE100 ESR spectrometer operating at X-band frequencies, having a $100 \mathrm{kHz}$ field modulation to obtain a first derivative EPR spectrum. DPPH with a $g$ value of 2.0036 was used for $g$-factor calculations. Angular variations were made at room temperature by rotating the crystal along the three mutually orthogonal axes $a, b$ and $c$ (see below).

\section{Crystal structure}

$\mathrm{CPPH}$ belongs to the series of struvite analogs, $\mathrm{CdMPO}_{4} \cdot 6 \mathrm{H}_{2} \mathrm{O}$ with $\mathrm{M}=\mathrm{K}, \mathrm{Tl}, \mathrm{NH}_{4}$, or $\mathrm{Rb}$. The host lattice crystallizes in the orthorhombic space group $P m n 2_{1}$ with unit cell parameters, $a=6.873, b=6.160$ and $c=11.087 \AA$ and contains two molecules per unit cell ${ }^{17}$. The structure contains cadmium ions surrounded by six oxygen atoms of the water of hydration. The six Cd-O bond distances $(\AA)$ are 2.095, 2.103, 2.071, 2.042, $2 \cdot 071$ and 2.042. Similarly none of the $\mathrm{O}-\mathrm{Cd}-\mathrm{O}$ bond angels are 90 or $180^{\circ}$. The $\mathrm{PO}_{4}^{3-}$ group is held together by a network of hydrogen bonds. The crystal data also indicates that the packing of $\mathrm{Cd}(\mathrm{II}), \mathrm{K}(\mathrm{I})$ and $\mathrm{PO}_{4}^{3-}$ ions occur in planes approximately parallel to the (101) and (011) planes.

\section{Results and discussion}

A good single crystal of the proper axis is selected and mounted on to the goniometer with a particular axis (e.g. $a$ axis) and is inserted into the EPR cavity for room temperature measurements. A few EPR spectra, at two different orientation of the crystal in the $b c$ plane, recorded at room temperature are shown in figure 1. Generally, an eightline pattern is expected for a vanadyl impurity when the applied magnetic field is parallel to a crystallographic axis, since the nuclear spin of vanadium is $7 / 2$ and all the magnetically different sites will coincide with the particular axis. Otherwise, in some cases, a set of resonance lines with very close hyperfine values can be seen. As the crystal is rotated, the lines split and then, one can observe more than eight lines. Hence, in the present case, in order to obtain the spin Hamiltonian parameters, the crystal was rotated for every 10 degrees in the three orthogonal planes and the isofrequency plots were plotted from the resonance lines. However, as expected in most of the vanadyl systems, the isofrequency plots were nearly similar in $b c$ and $a c$ planes, whereas the resonance lines are almost invariant in the $a b$ plane of rotation. This clearly indicates the axially symmetric nature of the $\mathrm{VO}$ (II) impurity in the lattice. When the crystal is rotated in the 
$b c$ plane, we notice two sets of eight lines, indicating the angular variation of the impurity, expected from the crystal structure of the host lattice. Figure 2 gives the angular variation of vanadium hyperfine lines in the $b c$ plane. Considering the nature of the isofrequency plots, one can assume without much error that the impurity is substitutionally incorporated into the host lattice in the place of $\mathrm{Cd}(\mathrm{II})$. This is also true if one considers the charges of various ions in the host lattice. As the maxima and minima of hyperfine resonances and $g$ values occur at the same angle, it implies that the principal

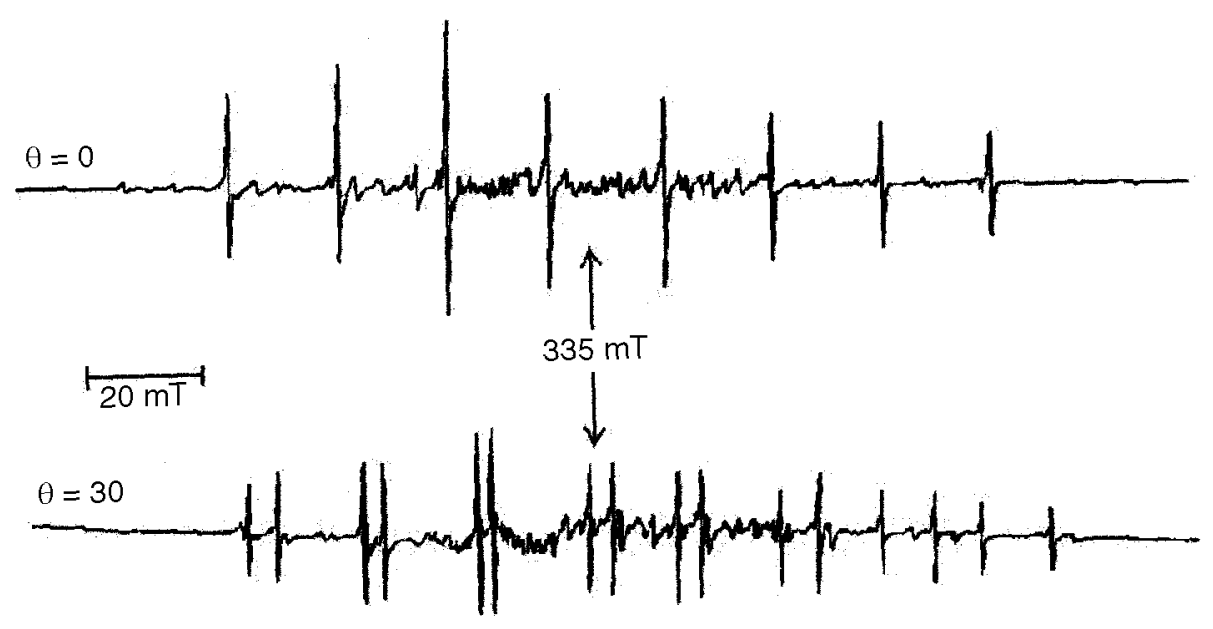

Figure 1. Single crystal room temperature EPR spectrum of VO(II)/CPPH at two different orientations of the crystal in $b c$ plane. Frequency $=9 \cdot 1324 \mathrm{GHz}$.

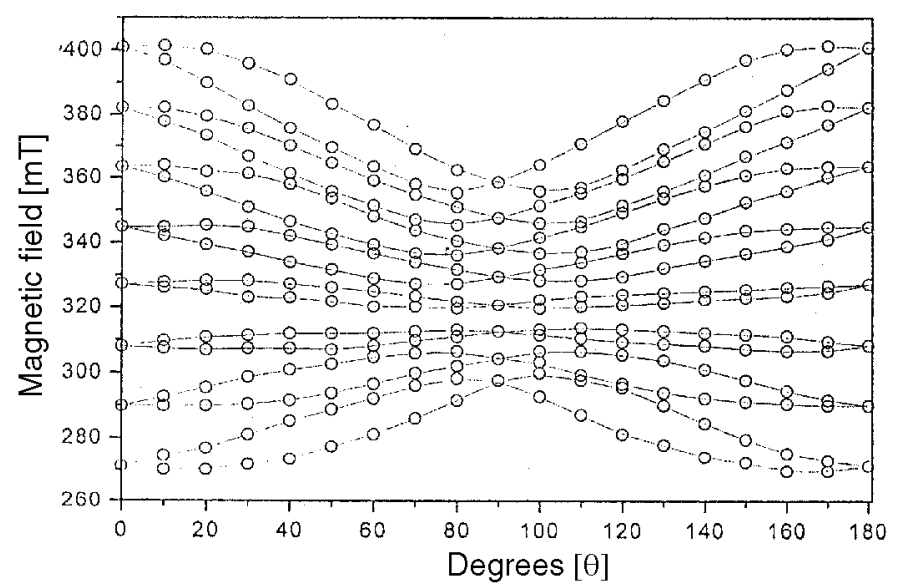

Figure 2. Isofrequency plot for VO(II)-doped CPPH in $b c$ plane of rotation. Similar plot was obtained in $a c$ plane whereas the resonance lines are invariant in $a b$ plane (see text). Frequency $=9 \cdot 1324 \mathrm{GHz}$. 
axes of $g$ and $A$ tensors are coincident. The variation in resonance positions in $a c$ and $b c$ planes, as mentioned earlier, are identical within the linewidth. Also, very minimal change in line positions is observed in the $a b$ plane. The spin-Hamiltonian parameters obtained from the isofrequency plot, using the standard procedure ${ }^{18}$, are given in table 1 , along with few more data from literature. As was mentioned in the case of $\mathrm{VO}$ (II) in $\mathrm{K}_{2} \mathrm{Zn}\left(\mathrm{SO}_{4}\right)_{2} \cdot 6 \mathrm{H}_{2} \mathrm{O}$, we also tried to compare the direction of the $\mathrm{VO}$ (II) impurity with those of metal-water bonds. The direction cosines of the impurity $\left(\theta=62 \cdot 9^{\circ}, \varphi=0.0^{\circ}\right)$ are somewhat closer to the direction cosines of the longest metal-water bond $\left(\theta=70 \cdot 0^{\circ}\right.$, $\varphi=5.0^{\circ}$ ), thus agreeing well with the reported data of Kasthurirengan and Soundararajan ${ }^{9}$ and Borcherts \& Kikuchi ${ }^{1}$. Here polar angle $\theta$ is defined as the angle of the applied magnetic field from the $z$-axis and azimuthal angle $\varphi$ as the angle from the $a$ axis in the $a b$ plane. According to them, the direction of principal $g$ value of highest intense line should lie along the direction of longest metal-water bond.

In order to confirm the spin Hamiltonian parameters obtained from single crystal data, the powder spectrum of $\mathrm{VO}(\mathrm{II}) / \mathrm{CPPH}$ was recorded at room temperature and is given in figure 3. Here, we can see only one set of eight parallel and perpendicular lines, confirming the strictly axial symmetric nature of the impurity. The spin-Hamiltonian parameters obtained from the powder spectrum are:

$$
g_{\|}=1.9340 ; g_{\perp}=1.9815, A_{\|}=19.78 \mathrm{mT} ; A_{\perp}=6.91 \mathrm{mT} .
$$

These values slightly differ from single crystal data, especially $A_{\perp}$ value. Hence, in order to confirm the powder data, the EPR spectrum of the powder sample was simulated using the program EPR-NMR ${ }^{19}$ with the data mentioned above. The agreement is very good. We have also simulated a few spectra at certain orientations using the single crystal data. Here also, we notice a fair amount of agreement. An interesting feature of the powder

Table 1. EPR parameters for vanadyl ions in a few selected diamagnetic host lattices.

\begin{tabular}{|c|c|c|c|c|c|}
\hline Host & $g_{\|}$ & $g_{\perp}$ & $A_{\|}(\mathrm{mT})$ & $A_{\perp}(\mathrm{mT})$ & Ref. \\
\hline Cadmium struvite & 1.931 & 1.993 & $18 \cdot 30$ & $7 \cdot 20$ & [11] \\
\hline $\mathrm{GeO}_{2}$ & 1.929 & 1.976 & $17 \cdot 55$ & $6 \cdot 82$ & [20] \\
\hline Apophyllite & 1.934 & 1.998 & $18 \cdot 34$ & $7 \cdot 24$ & [5] \\
\hline $\mathrm{CdNH}_{4} \mathrm{PO}_{4} \cdot 6 \mathrm{H}_{2} \mathrm{O}$ & 1.931 & 1.993 & $18 \cdot 30$ & $7 \cdot 20$ & [12] \\
\hline $\mathrm{ZnKPO}_{4} \cdot 6 \mathrm{H}_{2} \mathrm{O}$ & 1.936 & 1.976 & $20 \cdot 10$ & $7 \cdot 60$ & [21] \\
\hline $\mathrm{ZnNH}_{4} \mathrm{PO}_{4} \cdot 6 \mathrm{H}_{2} \mathrm{O}$ & 1.929 & 1.979 & $20 \cdot 00$ & 8.00 & {$[22]$} \\
\hline $\mathrm{K}_{2} \mathrm{Zn}\left(\mathrm{SO}_{4}\right)_{2} \cdot 6 \mathrm{H}_{2} \mathrm{O}$ & 1.932 & $2 \cdot 006$ & $19 \cdot 4$ & $7 \cdot 5$ & [9] \\
\hline $\mathrm{K}_{2} \mathrm{Mg}\left(\mathrm{SO}_{4}\right)_{2} \cdot 6 \mathrm{H}_{2} \mathrm{O}$ & 1.936 & $2 \cdot 003$ & $19 \cdot 6$ & $7 \cdot 4$ & [9] \\
\hline \multicolumn{6}{|l|}{ MPSH } \\
\hline Site 1 & 1.954 & 1.998 & $19 \cdot 80$ & $7 \cdot 61$ & [15] \\
\hline Site 2 & 1.993 & $1 \cdot 952$ & $7 \cdot 66$ & $19 \cdot 85$ & [15] \\
\hline \multicolumn{6}{|l|}{ MAPH } \\
\hline Site 1 & 1.941 & 1.994 & $19 \cdot 23$ & $7 \cdot 14$ & [16] \\
\hline Site 2 & 1.946 & 1.997 & $19 \cdot 08$ & $7 \cdot 29$ & [16] \\
\hline CPPH & 1.943 & 1.989 & $18 \cdot 45$ & $8 \cdot 71$ & Present study \\
\hline
\end{tabular}




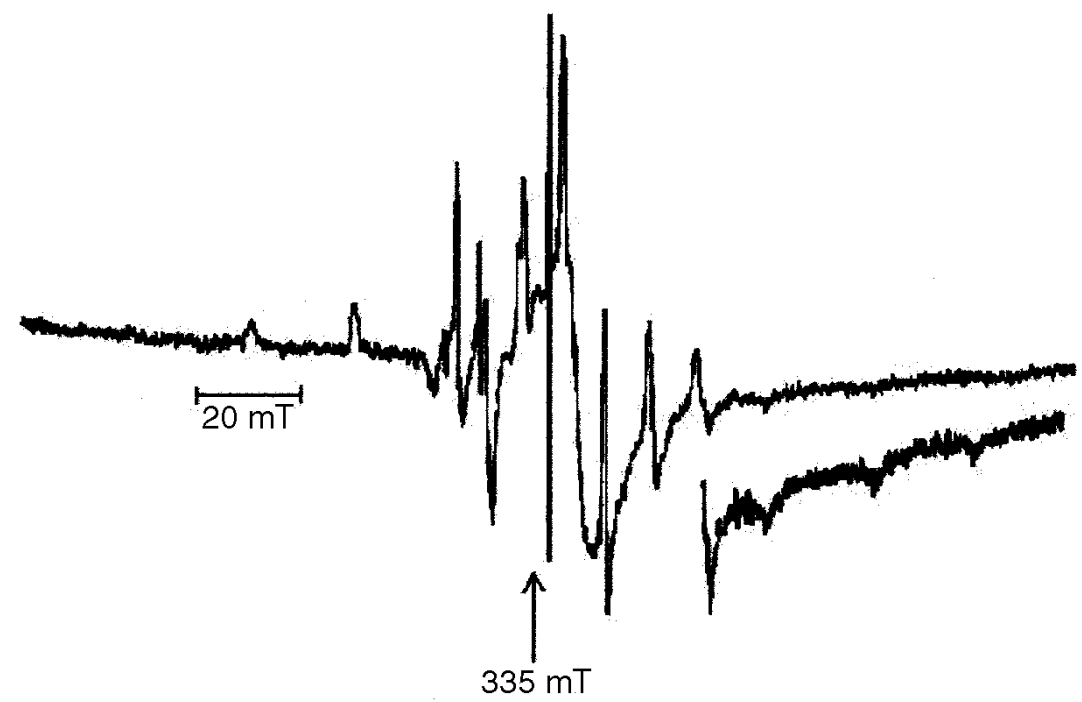

Figure 3. EPR spectrum of powdered sample of VO(II)/CPPH recorded at room temperature. Frequency $=9 \cdot 4276 \mathrm{GHz}$.

spectrum is the appearance of only two parallel resonance lines (the third one is very weak and can be seen at higher gains) on the high field side. As far as we are aware, a typical powder EPR spectrum of a vanadyl impurity consists of two parallel lines on low field side, a bunch of lines due to the overlap of three parallel and eight perpendicular lines, followed by three parallel lines. Our spectrum shows difference from the normal behaviour. When the EPR spectrum is recorded at higher amplitudes, the eighth line is noticed. A similar observation was noticed in yet another system reported from our laboratory ${ }^{16}$.

We have also calculated the admixture coefficients from the spin-Hamiltonian parameters. If $C_{1}, C_{2}$ and $C_{3}$ are the admixture coefficients, where the ground state $d_{x y}$ can mix respectively with $d_{x^{2}-y^{2}}, d_{y z}$ and $d_{x y}$, these are related to the spin Hamiltonian parameters by the relations ${ }^{10}$

$$
g_{\|}=2\left(3 C_{1}^{2}-C_{2}^{2}-2 C_{3}^{2}\right) ; \quad g_{\perp}=4 C_{1}\left(C_{2}-C_{3}\right) .
$$

These equations along with the normalization condition $\left(C_{1}^{2}+C_{2}^{2}+C_{3}^{2}=1\right)$ can be solved iteratively to obtain the admixture coefficients. The values thus obtained are given in table 2, along with the data reported for other systems. Using these coefficients and spin-Hamiltonian parameters, two more parameters $\kappa$ (a dimensionless constant describing core $s$-polarization) and $P$ have also been calculated using the equations,

$$
\begin{aligned}
& A_{\|}=P\left\{(-4 / 7)-\kappa+\left(\mathrm{g}_{\|}-g_{\mathrm{e}}\right)+(3 / 7)\left(g_{\perp}-g_{\mathrm{e}}\right)\right\}, \\
& A_{\perp}=P\left\{(2 / 7)-\kappa+(11 / 14)\left(g_{\perp}-g_{\mathrm{e}}\right)\right\} .
\end{aligned}
$$


Table 2. Orbital admixture coefficients and bonding parameters for a few vanadyl ions.

\begin{tabular}{lcccccc}
\hline Host & $C_{1}$ & $C_{2}$ & $C_{3}$ & $\kappa$ & $P \times 10^{-4} \mathrm{~cm}^{-1}$ & Ref. \\
\hline Apophyllite & 0.7018 & 0.7124 & 0.0529 & 0.86 & 122.7 & {$[5]$} \\
MPSH & 0.7000 & 0.7130 & 0.0404 & 0.85 & 133.1 & {$[15]$} \\
MPPH & 0.7014 & 0.7128 & 0.0067 & 0.83 & 130.7 & {$[23]$} \\
MAPH & 0.7019 & 0.7111 & 0.0400 & 0.91 & $112 \cdot 0$ & {$[16]$} \\
CPPH & 0.7015 & 0.7115 & 0.0016 & 0.77 & 141.9 & Present study \\
\hline
\end{tabular}

MPSH - magnesium potassium sulphate hexahydrate

$\mathrm{MPPH}$ - magnesium potassium phosphate hexahydrate

The values of $P$ and $\kappa$, obtained form the above equations are also given in table 2 . The standard value of $P$ for a free ion is $0.016 \mathrm{~cm}^{-1}$. The calculated value of $P$ in the present system is $0.0142 \mathrm{~cm}^{-1}$, which is considerably reduced to indicating a fair amount of covalent bonding, around $89 \%$, in the complex.

\section{Conclusions}

The previous studies of VO(II) indicate that the behaviour of vanadyl ion in a doped matrix is highly unpredictable, as regards its location. The impurity may enter substitutional, interstitial or both. Our current study indicates that the impurity has entered only one site, that too, the substitutional one. The spin Hamiltonian parameters have been derived from single crystal data. The principal value of the $g$ matrix is along the direction of longest metal-oxygen bond. An interesting observation is the pattern of powder spectrum. An extra insight is necessary to observe this kind of behaviour only in a few systems. The calculated admixture coefficients follow the normal trend. Other paramagnetic ions in this host lattice may throw some more light on understanding the effect of the host lattice indicating the various spin Hamiltonian parameters.

\section{Acknowledgements}

The authors thank the Council of Scientific \& Industrial Research, the Department of Science \& Technology, the University Grant Commission, New Delhi and the All India Council for Technical Education for financial assistance.

\section{References}

1. Borcherts R A and Kikuchi C 1964 J. Chem. Phys. 40227

2. Sathanarayana N and Radhakrishna S 1985 Cryst. Latt. Def. Amorph. Mat. 11113

3. Somesekharam V, Sivaprasad P, Ramesh K and Reddy Y P 1986 Phys. Scripta 33169

4. Viswanath A 1977 J. Chem. Phys. 673744 and references therein

5. Geetha Ramakrishnan, Swamy M B V L N, Sambasiva Rao P and Subramanian S 1991 Proc. Indian Acad. Sci. (Chem. Sci.) 103613

6. Ravikumar R V S S N, Jamalaiah B C, Chandrasekhar A V, Reddy B J, Reddy Y P and Sambasiva Rao P 1999 J. Alloys Compounds 84287

7. Umesh Gangadharmath B, Satish Annigeri M, Anil Naik D, Revankar K and Vinayak Mahale B 2001 J. Mol. Struc. 61572

8. Ravikumar R V S S N, Rajagopal Reddy V, Chandrasekhar A V, Reddy B J, Reddy Y P and Sambasiva Rao P 2002 J. Alloys Compounds 337272 
9. Kasthurirengan S and Soundararajan S 1975 J. Magn. Reson. 19357

10. Misra S K and Wang C 1989 Physica B159 321

11. Seth V P, Yadav S K and Jain V K 1983 Pramana - J. Phys. 2165

12. Ravikumar R V S S N, Madhu N, Reddy B J, Reddy Y P and Sambasiva Rao P 1997 Phys. Scripta $\mathbf{5 5} 637$

13. Satyanarayana N 1985 Spectrochim. Acta A41 1185

14. Kalkan H and Koksal F 1998 Solid State Communications 105307

15. Anandalakshmi H, Rajendiran T M, Venkatesan R and Sambasiva Rao P 2000 Spectrochim Acta, A56 2617 (Part 4)

16. Sougandi I, Venkatesan R and Sambasiva Rao P (submitted)

17. Mathew M and Schroeder L W 1979 Acta B35 11

18. Schonland D 1959 Proc. Phys. Soc. 73788

19. EPR-NMR Program developed by Clark F, Dickson R S, Fulton D B, Isoya J, Lent A, McGavin D G, Mombourquette M J, Nuttall R H D, Rao P S, Rinneberg H, Tennant W C and Weil J A, University of Saskatchewan, Saskatoon, Canada 1996

20. Siegel I 1964 Phys. Rev. A193 134

21. Ravikumar R V S S N, Madhu N, Chandrasekhar A V, Reddy B J, Reddy Y P, Sambasiva Rao P, Rajendiran T M and Venkatesan R 2001 Spectrochim. Acta A57 2789

22. Chand P and Agarwal O P 1991 Spectrochim. Acta A47 775

23. Poonguzhali R, Venkatesan R, Rajendiran T M, Rao P S, Ravikumar R V S S N and Reddy Y P 2000 Cryst. Res. Technol. 120335 